\title{
Effects of Weight Approximation Methods on Performance of Digital Beamforming Using Least Mean Squares Algorithm
}

\author{
Pranav Wani ${ }^{1}$, Amalendu Patnaik ${ }^{2}$ \\ ${ }^{I}$ B.Tech Student, IIT Roorkee, India \\ ${ }^{2}$ Assistant Professor, IIT Roorkee, India
}

\begin{abstract}
Adaptive Arrays are currently the subject of extensive research, for improving the quality of the receiving signals in the presence of interfering signals in wireless communications. In this paper beamforming is performed considering a digital system using Least Mean Squares algorithm. The main emphasis is to discuss the effects of the weight approximation procedures on the overall performance of the LMS algorithm and the Beamforming. The same results can be obtained for other adaptive beamforming algorithms and their various modifications available in the literature. In this paper Least Mean Squares Algorithm has been used to show the effects of weight approximation methods. Weight approximation methods are used to convert the continuous valued weights into discrete valued weights which can be used by digital systems for beamforming. Two algorithms have been proposed for weight approximation. Their performance has been compared with the conventional methods. Comparisons have been made for Beampattern, Sidelobe level, Convergence rate, Stability. The proposed algorithms have been observed to give overall better results for slight increase in the computational cost. The effect of the convergence coefficient $\boldsymbol{\mu}$ on the sidelobe levels of beampattern has also been discussed.
\end{abstract}

Keywords - Digital Beamforming, Least Mean Squares Algorithm, Phased Array Antenna, Weight Approximation

\section{INTRODUCTION}

An adaptive array [1] can be best described as a collection of sensors, feeding a weighting and summing network, with automatic signal dependent weight adjustments to emphasis the desired signal. With the direction of the incoming signals known or estimated, the next step is to use spatial processing techniques to improve the reception performance of the receiving antenna array based on this information. Some of these spatial processing techniques to improve the SNR are referred to as beamforming. Given a one dimensional linear array of elements and an impinging wavefront from an arbitrary point source, the directional power pattern $\mathrm{P}(\theta)$ can be expressed as

$$
P(\theta)=\int a(x) e^{-j \beta d(x, \theta)} d x
$$

Where $\mathrm{a}(x)$ is the amplitude distribution along the array, B is the phase constant, and $\mathrm{d}(x, \theta)$ is the relative distance the impinging wavefront, with an angle of arrival theta, has to travel between points uniformly spaced a distance $x$ apart along the length of the array.

There are several beamforming techniques available such as LMS, RMS, MVDR Beamforming, Classical Beamformer etc [1]. With the advancement in the integrated digital systems, formation of beam is being controlled by digital systems. Thus the selection of magnitude and phase of weights are being controlled by digital systems. Digital systems have many benefits, most significantly the small space requirements, low cost, very high speed. Thus in the backdrop of this it becomes important to investigate the various factors that control the digital beamforming.

The main focus of this paper is to investigate the methods of converting the continuous weights to discrete weights which can be used by digital systems.

\subsection{Introduction}

\section{Least Mean Squares Algorithm}

If sufficient knowledge of the desired signal is available, a reference signal $d$ can then be generated. These reference signals are used to determine the optimal weight vector wMSE $=[W 1, W 2, \ldots \ldots \ldots, W n]$. This is done by minimizing the mean square error of the reference signals and the output of the N-element antenna array.

The concept of reference signal use in adaptive antenna system was first introduced by Widrow where he described several pilot-signal generation techniques. One of the proposed techniques was a two mode adaption process whereby the transmitter alternated between sending a known pilot signal and actual data. The receiver had the knowledge of the pilot signal and used it as the desired response for the LMS adaptive 
algorithm. During actual data transmission, adaption would be switched off and the weights would coast until the pilot signal was turned back on. While this technique was never actually constructed, it provided the necessary impetus.

For beamforming considerations, the reference signal is usually obtained by a periodic transmission of a training sequence, which is a priori known at the receiver and is referred to as temporal reference. Information about the direction of the signal of interest is usually referred to as spatial reference.

The LMS algorithm [1] is probably the most widely used adaptive filtering algorithm, being employed in several communication systems. It has gained popularity due to it's low computational complexity and proven robustness. It incorporates new observations and iteratively minimizes linearly the mean square error. The LMS[1] algorithm changes the weight vector $w$ along the direction of the estimated gradient based on the negative steepest descent method. By the quadratic characteristics of the mean square error function that has only one minimum, the steepest descent is guaranteed to converge. The LMS [1] algorithm updates the weight vector according

$$
\begin{aligned}
w(k+1) & =w(k)-\frac{\mu}{2} \frac{\partial J_{w, w^{*}}}{\partial J_{w^{*}}} \\
& =w(k)+\mu e^{*}(k) x(k)
\end{aligned}
$$

Where, the rate of change of the objective function $\mathrm{Jw}_{\mathrm{W}} \mathrm{w}^{\prime}=|\mathrm{e}(\mathrm{k})|^{\wedge} 2$ has been derived earlier in and $\boldsymbol{\mu}$ is a scalar constant which controls the rate of convergence and stability of the algorithm. In order to guarantee stability in the mean-squared sense, the step size $\boldsymbol{\mu}$ should be restricted in the interval

$$
\begin{gathered}
0<\mu<2 / \lambda_{\max } \\
\lambda_{\max } \leq \operatorname{trace}\left\{R_{x x}\right\} \\
0<\mu<\frac{2}{\sum_{i=1}^{N} E\left\{x_{i}^{2}\right\}}
\end{gathered}
$$

Here $\mathrm{N}$ is the number of elements in the array. $\boldsymbol{\mu}$ is the convergence coefficient. It determines the convergence and stability of beampattern of the antenna. The algorithm can be stated as:

$$
\begin{gathered}
e(k)=d(k)-w^{H}(k) x(k) \\
w(k+1)=w(k)+\mu e^{*}(k) x(k)
\end{gathered}
$$

Beampattern of a 6-element linear array using LMS algorithm is shown below. The beam has been formed at $40^{0}$.

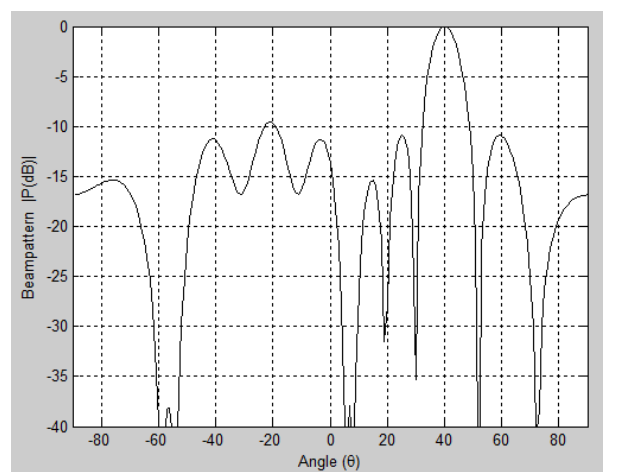

Fig 2.1: Beamforming using recursive Least Mean Squares algorithm for a 6-element uniform linear array. Reference signal is at $40^{\circ}$

\subsection{Digital Beamforming using LMS Algorithm}

While forming the beampattern using LMS algorithm, the weights are iteratively updated. The magnitude and the phase of the weights are provided to the antenna elements using analog systems. With the advances in the integrated digital systems, it has become more convenient to provide the required magnitude and phase to the elements digitally. Since there exists a limitation to the values that can be provided using digital systems, it becomes important to study the methods to convert the analog values to the digital values so as to obtain the acceptable output of the antennas. In this report two available methods for the conversion of contentious values to discrete values have been mentioned. Further two new methods have been suggested. It 
has been observed that the two new methods provide significant improvement over the available methods in terms of antenna beampattern, sidelobe levels and small improvement over convergence rate.

\subsection{Importance of Convergence Coefficient}

LMS algorithm depends on the continuous upgradation of the weights to get the desired beam in a particular direction. The convergence rate of the algorithm depends on the convergence coefficient $\boldsymbol{\mu}$. Larger coefficient ensures larger steps, but it comes at the cost of stability. Smaller coefficients make the step size smaller, but the deviation is also small, which compensates for the small step size. Thus too large or too small $\boldsymbol{\mu}$ tends to give slow convergence. There is a optimum value of $\boldsymbol{\mu}$ which provides the fastest convergence. value depends on

Thus the maximum convergence rate is obtained at a particular optimum value of $\mu$. This optimum

$\rightarrow$ The design of the array

$\rightarrow$ The environmental condition

(Environmental conditions include the noise present in the environment.)

To illustrate the effect of convergence coefficient $\boldsymbol{\mu}$ on the convergence rate a 6-element uniform linear array has been considered. Beam has been formed using LMS algorithm. Error plots are shown below for three values of $\boldsymbol{\mu} ; 0.1,0.2$, and 0.3 .

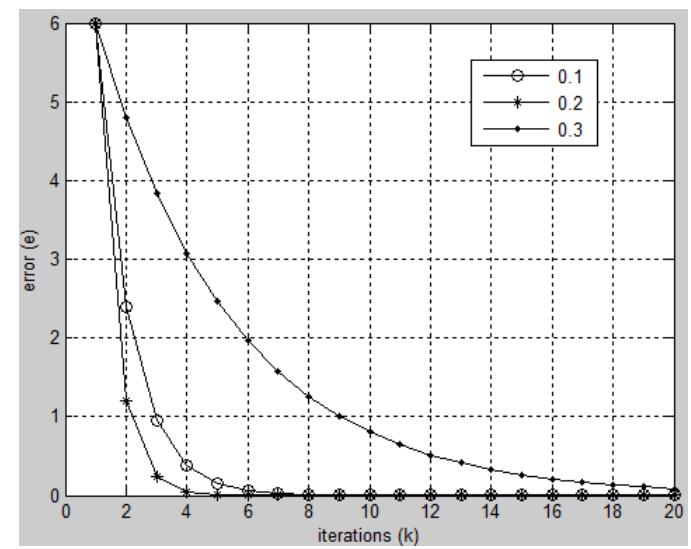

Fig 2.2: Error plots for different values of $\boldsymbol{\mu}$ using Least Mean Squares Algorithm

\section{Conversion Of Analogous Weights To Digital Weights 3.1 Conventional Algorithms to convert Continuous Valued Weights to Discrete Valued Weights}

a) O-Floor Method

This method is most basic way to determine the value of any number available in continuous scale for the use in digital systems, and is widely used [2]. In this method the number is approximated to the nearest available number to the left of it. Continuous weights are converted to discrete values by shifting the decimal numbers to the nearest left integer. The LMS algorithm can thus be written as:

$$
\begin{gathered}
e(k)=d(k)-w^{H}(k) x(k) \\
w(k+1)=w(k)+\mu e^{*}(k) x(k) \\
f=\text { floor }\left[\frac{w(k+1)}{\text { step }}\right] \\
w(k+1)=f * \text { step }
\end{gathered}
$$

Here ' $w$ ' is the value of weight in continuous scale. 'Step' is the step size of the available digital system. It is the minimum distance between two nearest numbers that the system can provide. Step is the resolution of the digital system.

\section{b) 0.5 -Floor Method}

This method is another available method to convert the analog values to the digital [2]. In this method the number is approximated to the nearest available value. It may thus be shifted either to the left or 
right depending upon it's location between the two nearest available values. The LMS algorithm can thus be given as: step is the step size.

$$
\begin{gathered}
e(k)=d(k)-w^{H}(k) x(k) \\
w(k+1)=w(k)+\mu e^{*}(k) x(k) \\
w(k+1)=w(k+1)+\frac{\text { step }}{2} \\
f=\text { floor }\left(\frac{w(k+1)}{s t e p}\right) \\
w(k+1)=f * \text { step }
\end{gathered}
$$

\subsection{Improved Algorithms for converting Continuous valued Weights into Discrete Valued Weights}

a)Minimum Modulus Method

This is one of the two methods that have been proposed. After simulations it can be observed that this method provides better results compared to the available methods.

In this method the value of the weights are selected to the nearest value available in the left or in the right on the number line depending on the value of the modulus of the output. Thus a decimal number would be shifted to the nearest value available in the left if the number if positive. Similarly, a number would be shifted to the nearest value available in the right of the number scale, if the number is negative.

Thus the LMS algorithm can be written as:

$$
\begin{gathered}
e(k)=d(k)-w^{H}(k) x(k) \\
w(k+1)=w(k)+\mu e^{*}(k) x(k) \\
w(k+1)=w(k+1) ; \text { if } w \geq 0 \\
w(k+1)=w(k+1)+\text { step } ; \text { if } w<0 \\
f=\text { floor }\left[\frac{w(k+1)}{\text { step }}\right] \\
w(k+1)=f * \text { floor }
\end{gathered}
$$

b)Motion Corroboration Method

In this method the value of the weights is approximated depending on the present value of the weights as well as the previous values of the weights. The values are approximated so as to corroborate the direction of motion of the weights. Thus if a number in the present iteration has shifted right with respect to it's position in previous iteration, then the number will be approximated to it's nearest value available in the right. Similarly, if a number has shifted left in it's present iteration compared to it's position in the previous iteration, then the number will be approximated to the nearest available value to it's left.

Thus the LMS algorithm using motion corroboration algorithm for weight approximation can be written as: step is the step size, and $e$ is the error.

$$
\begin{gathered}
e(k)=d(k)-w^{H}(k) x(k) \\
w(k+1)=w(k)+\mu e^{*}(k) x(k) \\
w(k+1)=w(k+1)+\text { step } ; \text { if } e \geq 0 \\
w(k+1)=w(k+1) ; \text { if } e<0 \\
f=\text { floor }\left(\frac{w(k+1)}{\text { step }}\right) \\
w(k+1)=f * \text { step }
\end{gathered}
$$

\section{Experimental Simulations}

We have used a 10-element linear array. Beam has been formed using Least Mean Squares algorithm. The reference signal has been taken at $40^{\circ}$. The step size 'step' used is 0.01 . For weight approximation, above discussed four algorithms have been used. 
For every weight approximation algorithm, the optimum $\boldsymbol{\mu}$ has been calculated using Particle Swarm Optimization Algorithm (PSO) to get the minimum sidelobe level. Minimum sidelobe level is obtained for $\boldsymbol{\mu}$ which corresponds to the fastest convergence rate. Thus $\boldsymbol{\mu}$ has been optimized to give fastest convergence rate. Another method of optimizing $\boldsymbol{\mu}$ was that for lowest error. But the value of $\boldsymbol{\mu}$ giving lowest average error does not give lowest sidelobe level. This result has been shown through simulation in section 5 .

Thus for this simulation and for all the other simulations presented in this article, $\boldsymbol{\mu}$ has been optimized for every weight approximating algorithm, to give the fastest convergence rate. This ensures the minimum sidelobe level for every algorithm.

After optimization, the various values of $\boldsymbol{\mu}$ that have been used in this example are $\boldsymbol{\mu}=0.1148$ for 0 -floor method (fig 4.1a), $\boldsymbol{\mu}=0.1151$ for 0.5 -floor method (fig 4.1b), $\boldsymbol{\mu}=0.1124$ for minimum modulus method (fig 4.1c), $\boldsymbol{\mu}=0.1245$ for motion corroboration method (fig 4.1d).

For this example and for all other examples sidelobe level has been represented in $\mathrm{dB}$ (decibels), and beamwidth in degrees.

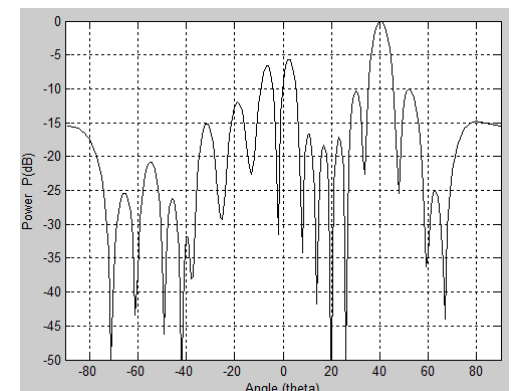

(a)

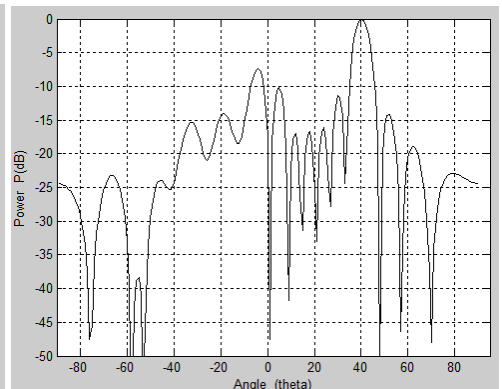

(b)

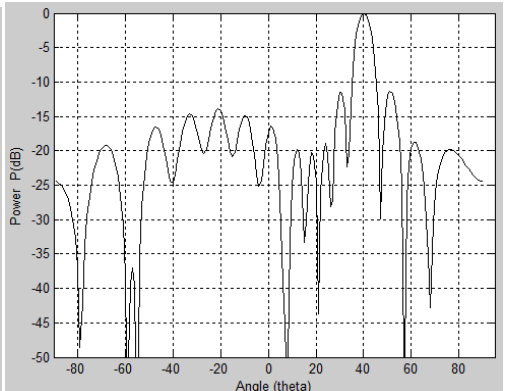

(c)

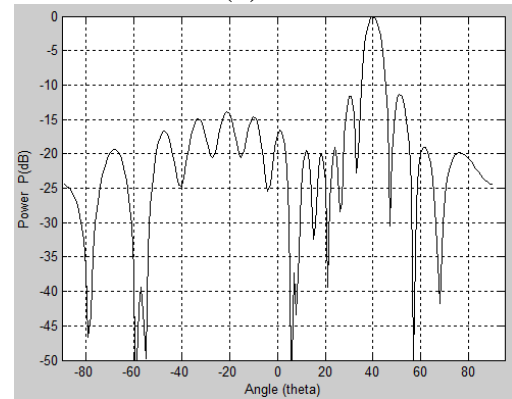

(d)

Fig 4.1: Digital beamforming using LMS algorithm. Methods used for weight approximation are (a) 0-floor method, (b) 0.5 floor method, (c) minimum modulus method, (d) motion corroboration method

\begin{tabular}{|l|c|c|}
\hline $\begin{array}{l}\text { Weight Approximation } \\
\text { Method }\end{array}$ & $\begin{array}{l}\text { Max. Sidelobe } \\
\text { Level }\end{array}$ & Beamwidth \\
\hline & & \\
\hline 1. 0-Floor Method & -5.6397 & 6.490 \\
\hline 2. 0.5-Floor Method & -7.4027 & 6.640 \\
\hline $\begin{array}{l}\text { 3. Minimum Modulus } \\
\text { Method }\end{array}$ & $\mathbf{- 1 3 . 8 3 6 7}$ & $\mathbf{6 . 2 9 4}$ \\
\hline $\begin{array}{l}\text { 4. Motion Corroboration } \\
\text { Method }\end{array}$ & $\mathbf{- 1 3 . 8 6 8 3}$ & $\mathbf{6 . 2 9 8}$ \\
\hline
\end{tabular}

Fig 4.2: Sidelobe levels and Beam widths for beam patterns in fig. 4.1

As an another example, a 30-element uniform linear array has been used for forming beam using LMS algorithm. Reference signal has been taken at $40^{\circ}$. Different methods have been used for converting the continuous valued weights to discrete ones. The step size step has been taken to be 0.005 . The plots are shown below.

The various values of optimum $\boldsymbol{\mu}$ used for various weight approximating algorithms are $\boldsymbol{\mu}=0.0334$ for 0-floor method (fig 4.3a), 0.5-floor method (fig 4.3b), and motion corroboration method (fig 4.3d), and $\boldsymbol{\mu}=0.0336$ for minimum modulus method (fig $4.3 \mathrm{c}$ ). 


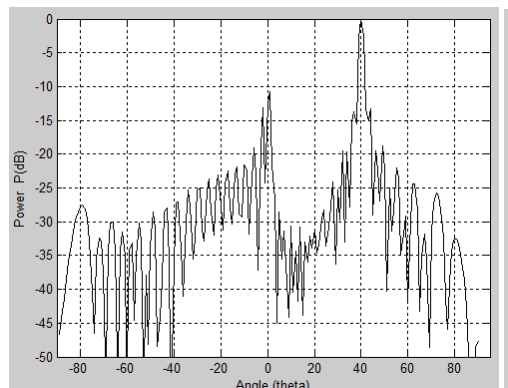

(a)

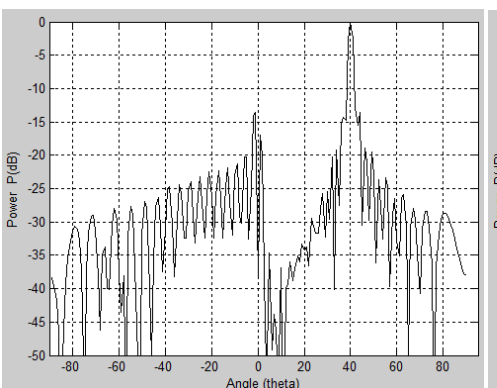

(b)

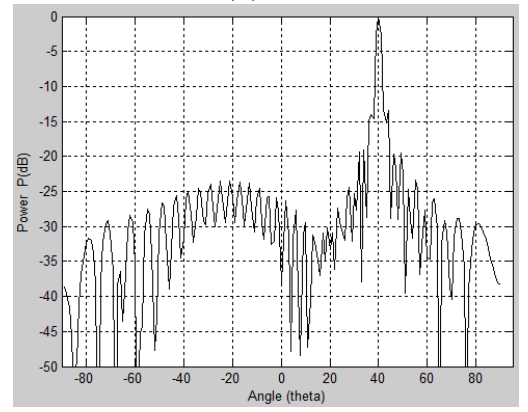

(d)

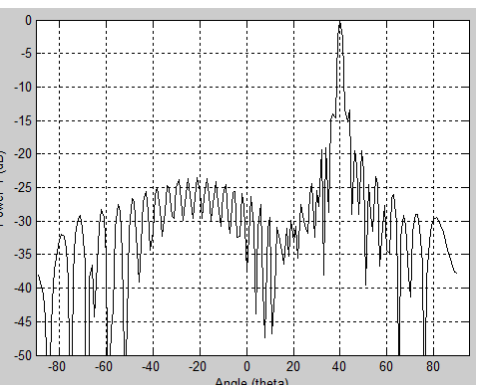

(c)

Fig 4.3: Digital beamforming using LMS algorithm. Methods used for weight approximation are (a) 0 -floor method, (b) 0.5-floor method, (c) minimum modulus method, (d) motion corroboration method

\begin{tabular}{|l|c|c|}
\hline $\begin{array}{l}\text { Weight Approximation } \\
\text { Method }\end{array}$ & $\begin{array}{l}\text { Max. Sidelobe } \\
\text { Level }\end{array}$ & Beamwidth \\
\hline & -10.7315 & 2.1520 \\
\hline 1. 0-Floor Method & -13.6395 & 2.1680 \\
\hline 2. 0.5-Floor Method & -23.5340 & $\mathbf{2 . 1 6 8 0}$ \\
\hline $\begin{array}{l}\text { 3. Minimum Modulus } \\
\text { Method }\end{array}$ & $-\mathbf{2 3 . 5 4 7 7}$ & $\mathbf{2 . 1 6 8 0}$ \\
\hline $\begin{array}{l}\text { 4. Motion Corroboration } \\
\text { Method }\end{array}$ & \\
\hline
\end{tabular}

Fig 4.4: Sidelobe levels and Beam widths for beam patterns of fig. 4.3

As an another example, a 30-element uniform linear array is used for forming beam using LMS algorithm.3- elements have been assumed to be failed. Element no. 2, 8, 16 have been assumed to be failed. Failure has been assumed to be complete. Complete failure means that no signal is transmitted through the failed elements. Reference signal has been taken at $40^{\circ}$. Different methods have been used for converting the continuous valued weights to discrete ones. The plots are shown below. The step size step is taken to be 0.005 .

Various values of the convergence coefficients used in this example are $\boldsymbol{\mu}=0.0417$ for 0 -floor method (fig $4.5 \mathrm{a}$ ), $\boldsymbol{\mu}=0.0417$ for 0.5 -floor method (fig $4.5 \mathrm{~b}$ ), $\boldsymbol{\mu}=0.0419$ for minimum modulus method (fig $4.5 \mathrm{c}$ ), and $\boldsymbol{\mu}=0.0416$ for motion corroboration method (fig $4.5 \mathrm{~d}$ ).

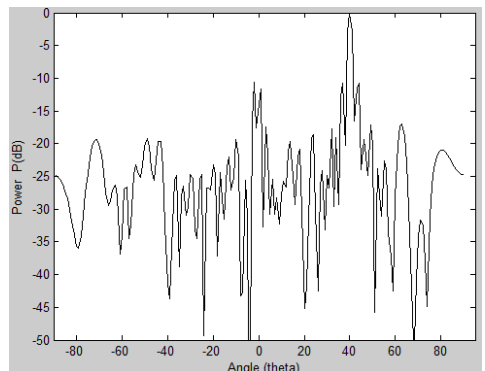

(a)

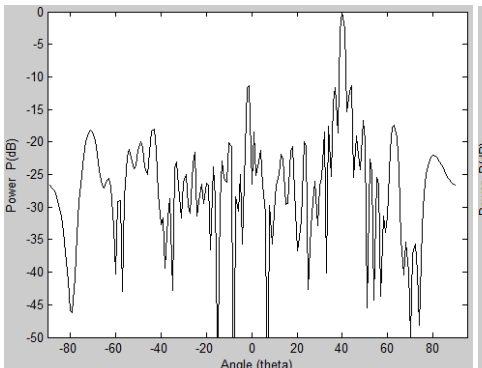

(b)

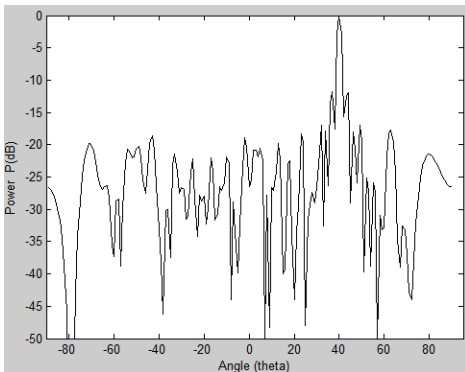

(c) 


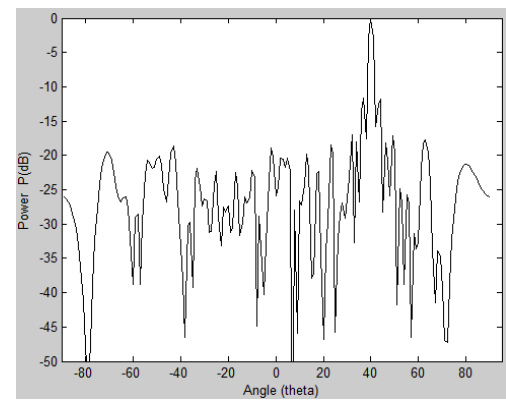

(d)

Fig 4.5: Digital beamforming using LMS algorithm. Methods used for weight approximation are (a) 0-floor method, (b) 0.5-floor method, (c) minimum modulus method, (d) motion corroboration method

\begin{tabular}{|l|c|c|}
\hline $\begin{array}{l}\text { Weight Approximation } \\
\text { Method }\end{array}$ & $\begin{array}{l}\text { Max. Sidelobe } \\
\text { Level }\end{array}$ & Beamwidth \\
\hline & -10.5745 & 2.0480 \\
\hline $\begin{array}{l}\text { 1. 0-Floor Method } \\
\text { 2. 0.5-Floor Method }\end{array}$ & -10.5745 & 2.0760 \\
\hline $\begin{array}{l}\text { 3. Minimum Modulus } \\
\text { Method }\end{array}$ & $\mathbf{- 1 8 . 3 8 0 2}$ & $\mathbf{2 . 0 8 8 0}$ \\
\hline $\begin{array}{l}\text { 4. Motion Corroboration } \\
\text { Method }\end{array}$ & $\mathbf{- 1 8 . 4 3 1 2}$ & $\mathbf{2 . 0 8 4 0}$ \\
\hline
\end{tabular}

Fig 4.6: Sidelobe levels and Beam widths for beam patterns of fig. 4.5

To plot the error plots, the same example mentioned above has been used. All the values of different parameters have been kept constant. The only change that has been made is to have 6 failed elements instead of 3 . The elements $2,8,16,22,26,30$ have been assumed to be failed. It is not a practical scenario to have 6 failed elements, but has been taken for illustration purpose only.

The weight approximation algorithms used are 0-floor method (fig 4.7a), 0.5-floor method (fig 4.7b), minimum modulus method (fig 4.7c), and motion corroboration method (fig 4.7d).

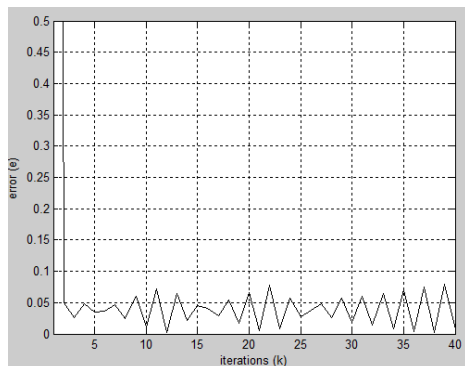

(a)

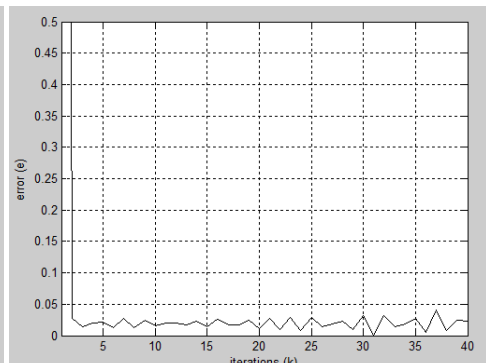

(b)

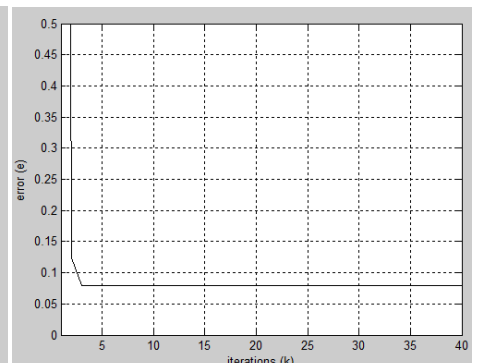

(c)

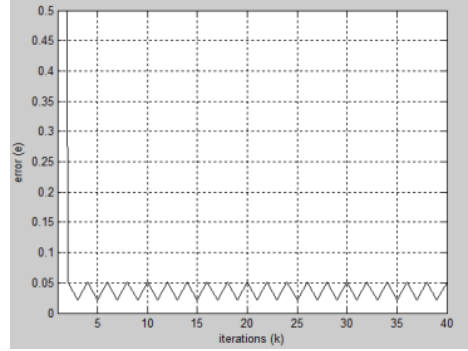

(d)

Fig 4.7: Convergence plots for LMS algorithm. Methods used for weight approximation are (a) 0 -floor method, (b) 0.5-floor method, (c) minimum modulus method, (d) motion corroboration method 


\begin{tabular}{|l|c|c|}
\hline $\begin{array}{l}\text { Weight Approximation } \\
\text { Method }\end{array}$ & $\begin{array}{l}\text { Max. Sidelobe } \\
\text { Level }\end{array}$ & Beamwidth \\
\hline & -10.2850 & 2.1140 \\
\hline $\begin{array}{l}\text { 1. 0-Floor Method } \\
\text { 2. 0.5-Floor Method }\end{array}$ & -9.3149 & 2.1460 \\
\hline $\begin{array}{l}\text { 3. Minimum Modulus } \\
\text { Method }\end{array}$ & $\mathbf{- 1 4 . 6 2 7 7}$ & $\mathbf{2 . 1 7 2 0}$ \\
\hline $\begin{array}{l}\text { 4. Motion Corroboration } \\
\text { Method }\end{array}$ & $\mathbf{- 1 4 . 4 4 8 4}$ & $\mathbf{2 . 1 6 6 0}$ \\
\hline
\end{tabular}

Fig 4.8: Sidelobe levels and Beam widths for convergence plots of fig. 4.7

From the above examples it can be observed that the two proposed algorithms, Minimum Modulus method and Motion Corroboration method perform significantly better that the 0 -floor method and 0.5 -floor method in terms of sidelobe level. The sidelobe levels obtained by the proposed methods are significantly lower for a small increment in the beamwidth, and computational cost.

The convergence rate of LMS algorithm using motion corroboration is the fastest among the four discussed algorithms. It can also be observed that the output of LMS using minimum variance method is the most stable.

\section{Efect Of Convergence Coefficient $\mu$}

In this section, we wish to find the optimum value of convergence coefficient $\boldsymbol{\mu}$ to get the lowest sidelobe levels using Least Mean Squares Algorithm. Here we are using digital beamformer. For the purpose of weight approximation we use motion corroboration algorithm.

For the purpose of simulation, a 6- element uniform linear array has been used. The step size step has been fixed to 0.1 . The reference signal is assumed to be at $40^{\circ}$. We have used five different values of $\boldsymbol{\mu}$. One of the values of $\boldsymbol{\mu}$ has been calculated by PSO for the condition of fastest convergence rate. The value of $\boldsymbol{\mu}$ found for fastest convergence rate was $\boldsymbol{\mu}=0.1423$. Another value of $\boldsymbol{\mu}$ was calculated using PSO for the condition of lowest steady state error. The value of $\boldsymbol{\mu}$ for lowest steady state error was found to be $\boldsymbol{\mu}=0.050$. The remaining three values were randomly taken to be in the vicinity of the two calculated values. The other three values of $\boldsymbol{\mu}$ that were used are $\boldsymbol{\mu}=0.0213,0.00803,0.0617$.

The error plots for the two calculated values, fastest convergence rate (0.1423), and lowest steady state error (0.050) are shown in fig 5.1. The beam patterns using all the five values of $\boldsymbol{\mu}$ are shown in fig 5.2.

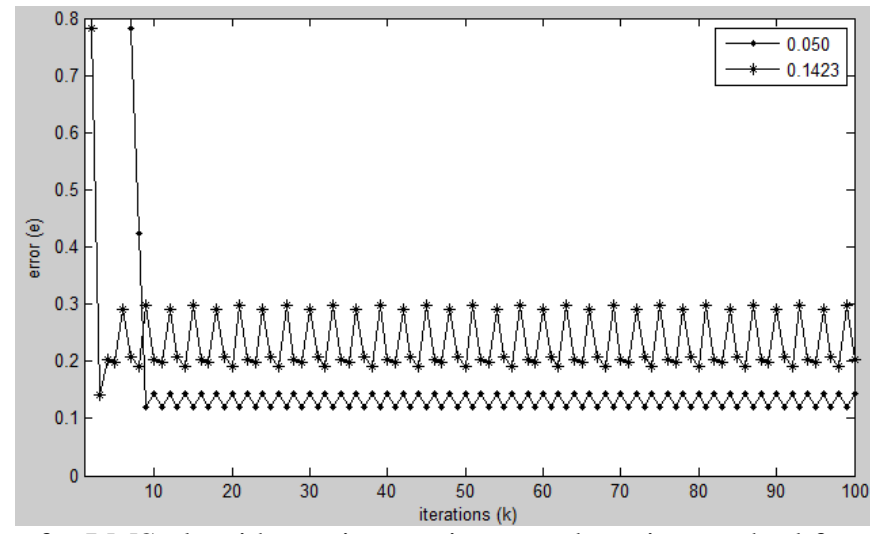

Fig 5.1: Convergence plot for LMS algorithm using motion corroboration method for weight approximation for two values of $\boldsymbol{\mu}(0.050$, and 0.1423$)$ 


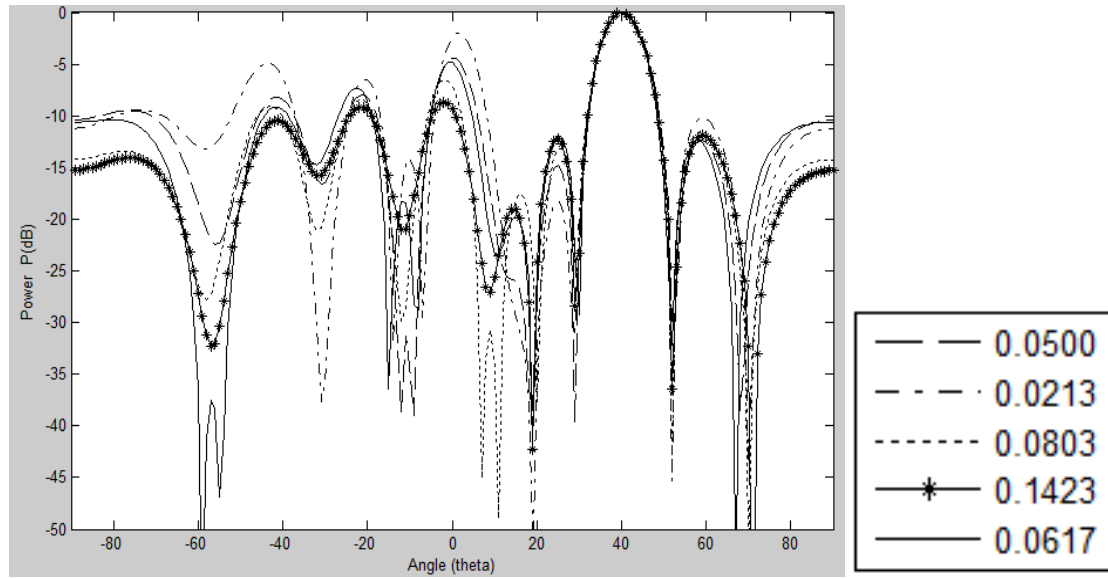

Fig 5.2: Digital Beamforming using LMS algorithm with motion corroboration as weight selection algorithm.

\begin{tabular}{|l|l|l|}
\hline $\begin{array}{l}\text { Convergence } \\
\text { Coefficient }\end{array}$ & $\begin{array}{l}\text { Max. Sidelobe } \\
\text { Level }\end{array}$ & Beamwidth \\
\hline 1. 0.05 & -4.4022 & 10.3920 \\
\hline 2. 0.0213 & -2.0201 & 10.2460 \\
\hline 3. 0.0803 & -6.4690 & 10.1280 \\
\hline 4. 0.1423 & -8.6421 & 10.4020 \\
\hline 5. 0.0617 & -4.8523 & 10.0280 \\
\hline
\end{tabular}

Fig 5.3: Sidelobe levels and Beam widths for beam patterns of fig. 5.2

From the above plots it can be clearly seen that the lowest sidelobe levels is obtained in LMS algorithm for the value of $\boldsymbol{\mu}$ that corresponds to the fastest convergence rate. Thus it can be concluded that the value of $\boldsymbol{\mu}$ corresponding to the fastest convergence rate, and not the one corresponding to the lowest steadystate error, results in the lowest sidelobe levels.

\section{Conclusion}

In this article digital beamforming using Least Mean Squares algorithm was discussed. The focus of this article was to discuss the effects of weight approximation algorithms on the beamforming algorithms. Approximately the same effects can be observed with various beamforming algorithms and their derivatives available in the literature. However in this article only basic LMS algorithm was considered.

In this article two new weight approximation methods were proposed. It was observed that the performance of the proposed algorithms were better than the available methods. The new proposed methods resulted in the significantly lower sidelobe levels and faster convergence rate at the rate of small increase in computational cost. In this article it was also shown that the lowest sidelobe levels are obtained for $\boldsymbol{\mu}$ corresponding to the fastest convergence rate and not necessarily to the $\boldsymbol{\mu}$ corresponding to the lowest steadystate error.

\section{REFERENCES}

[1] Constantine A. Balanis and Panayiotis I. Ioannides, Introduction to Smart Antennas, $1^{\text {st }}$ edition: Morgan \& Claypool, 2007

[2] Donald Hearn and M. Pauline Baker, Computer Graphics - C Version, Prentice Hall of India.

[3] J. Kennedy and R. C. Eberhart, Swarm Intelligence, San Francisco, CA: Morgan Haufmann, 2001 